\title{
Vitamin D deficiency, oxidative stress and antioxidant status: only weak association seen in the absence of advanced age, obesity or pre-existing disease
}

\author{
Erica W. Wang ${ }^{1}$, Parco M. Siu ${ }^{1}$, Marco Y. Pang ${ }^{2}$, Jean Woo ${ }^{3}$, Andrew R. Collins ${ }^{4}$ and Iris F. F. Benzie ${ }^{1 *}$ \\ ${ }^{1}$ Department of Health Technology and Informatics, The Hong Kong Polytechnic University, Yuk Choi Road, Hung Hom, \\ Kowloon, Hong Kong \\ ${ }^{2}$ Department of Rehabilitation Sciences, The Hong Kong Polytechnic University, Yuk Choi Road, Hung Hom, Kowloon, Hong Kong \\ ${ }^{3}$ Department of Medicine and Therapeutics, The Chinese University of Hong Kong, New Territories, Hong Kong \\ ${ }^{4}$ Department of Nutrition, The University of Oslo, PO Box 1072 Blindern, 0316 Oslo, Norway
}

(Submitted 10 April 2017 - Final revision received 26 June 2017 - Accepted 27 June 2017 - First published online 31 July 2017 )

\section{Abstract}

Vitamin D deficiency (plasma 25-hydroxycholecalciferol $(25(\mathrm{OH}) \mathrm{D})<50 \mathrm{nmol} / \mathrm{l})$ is highly prevalent, increases risk of non-communicable diseases (NCD) and associates with increased oxidative stress in obese subjects, the elderly and patients suffering from NCD. If confirmed as an independent driver of oxidative stress, nutritional and other public health strategies to improve vitamin D status would be strongly supported. We investigated vitamin $\mathrm{D}$ /oxidative stress links without the confounding effects of advanced age, obesity, smoking or pre-existing disease. Plasma 25(OH)D and biomarkers of oxidative stress and antioxidant status (plasma allantoin, oxidised LDL, ferric reducing antioxidant power (FRAP), ascorbic acid, urine 8-oxo-7,8-dihydro-2'-deoxyguanosine) were measured in fasting samples from 196 consenting, healthy adults aged 18-26 years. Correlation between $25(\mathrm{OH}) \mathrm{D}$ and each biomarker as well as biomarker differences across $25(\mathrm{OH}) \mathrm{D}$ quartiles and groups $(<25 / 25-49 / \geq 50 \mathrm{nmol} / \mathrm{l})$ were investigated. Median $25(\mathrm{OH}) \mathrm{D}$ was $40 \mathrm{nmol} / \mathrm{l} ;>70 \%$ of participants were vitamin D deficient. No significant correlations and no biomarker differences across 25(OH)D quartiles or groups were seen except for total antioxidant status. A weak direct association $(r 0.252, P<0.05)$ was observed between $25(\mathrm{OH}) \mathrm{D}$ and FRAP, and those in the lowest $25(\mathrm{OH}) \mathrm{D}$ quartile and group had significantly lower FRAP values. Results did not reveal a clear link between vitamin D status and oxidative stress biomarkers in the absence of advanced age, obesity and disease, though some evidence of depleted antioxidant status in those with vitamin D deficiency was seen. Poor antioxidant status may pre-date increased oxidative stress. Study of effects of correction of deficiency on antioxidant status and oxidative stress in vitamin D-deficient but otherwise healthy subjects is needed.

Key words: Vitamin D: 25-Hydroxycholecalciferol: Oxidative stress: Allantoin: 8-Oxo-7,8-dihydro-2'-deoxyguanosine: Oxidised LDL: Ferric reducing antioxidant power

Non-communicable diseases (NCD) contribute most to the huge and increasing burden of death and disability worldwide, and yet are largely preventable by healthy lifestyle and dietary choices ${ }^{(1)}$. Subtle, initially 'hidden' biomolecular changes, if left to develop and accumulate, lead to cellular dysfunction, tissue damage and, eventually, to $\mathrm{NCD}^{(1-5)}$. Many of these changes are linked to oxidative stress $^{(2-4)}$. Various factors that increase oxidative stress, including inflammation, smoking and obesity, are risk factors for $\mathrm{NCD}^{(1-5)}$. Identifying and correcting modifiable risk factors for NCD in still-healthy subjects would promote long-term health and have significant socioeconomic benefit for our ageing communities ${ }^{(1,5)}$. Deficiency of vitamin D (plasma 25-hydroxycholecalciferol $(25(\mathrm{OH}) \mathrm{D})<50 \mathrm{nmol} / \mathrm{l})$ is a suggested risk factor for NCD. This is based on experimental evidence of pleiotropic effects of vitamin $\mathrm{D}$ and results of population-based studies that show strong association between poor vitamin D status and development of $\mathrm{NCD}^{(6-22)}$. A detailed review of such studies is beyond the scope of this paper, but some examples are presented briefly. A Finnish study of $>1100$ men and women aged 53-73 years found a significantly higher risk of death in those with low vitamin D during an average follow-up of 9 years; hazard ratio (HR) for all-cause mortality was $2.06(95 \%$ CI $1.12,3.80)$ in the lowest compared with the highest tertile of $25(\mathrm{OH}) \mathrm{D}^{(15)}$. In the German population-based ESTHER study of almost 10000 subjects aged 50-74 years at baseline, overall mortality during follow-up (median 9.5 years) showed HR for all-cause death was significantly higher in those with vitamin $\mathrm{D}$ deficiency: in those with plasma $25(\mathrm{OH}) \mathrm{D}<30$ or $30-50 \mathrm{nmol} / \mathrm{l}$ the mortality HR values were, respectively, 1.71 (95\% CI $1.43,2.03)$ and 1.17 (95\% CI 1.02, 1.35) when compared with those with

Abbreviations: 25(OH)D, 25-hydroxycholecalciferol; 8-oxodG, 8-oxo-7,8-dihydro-2'-deoxyguanosine; FRAP, ferric reducing antioxidant power; NCD, noncommunicable diseases; OxLDL, oxidised LDL.

* Corresponding author: I. F. F. Benzie, fax +852 2362 4365, email htbenzie@polyu.edu.hk 
$25(\mathrm{OH}) \mathrm{D}>50 \mathrm{nmol} / \mathrm{l}$, and there were similar findings for death from CVD or cancer ${ }^{(7)}$. In a nested case-control study involving $>500000$ subjects across ten European countries, those in the lowest quintile of pre-diagnostic $25(\mathrm{OH}) \mathrm{D}$ concentration had a $40 \%(P<0.001)$ higher risk of incident colorectal cancer ${ }^{(16)}$. In a study of $>1000$ Korean subjects, incidence of type 2 diabetes at up to 5 years of follow-up was higher in those with vitamin D deficiency independently of other risk factors: hazard ratios in those with plasma $25(\mathrm{OH}) \mathrm{D} \leq 25$ or $>25<50 \mathrm{nmol} / 1$ were $3.23(95 \% \mathrm{CI}$ $1.66,6.30)$ and 2.06 (95\% CI 1.22, 3.49), respectively, compared with those with plasma $25(\mathrm{OH}) \mathrm{D} \geq 50 \mathrm{nmol} / \mathrm{l}^{(17)}$, and it is estimated that each $25 \mathrm{nmol} / 1$ increment in plasma $25(\mathrm{OH}) \mathrm{D}$ is associated with a $24 \%$ decrease in risk of 5 -year incidence of diabetes: OR $0.76(95 \% \text { CI } 0.63,0.92)^{(18)}$. However, not all studies agree, and it is noted that vitamin D deficiency could be a consequence rather than an underlying cause of disease ${ }^{(23)}$. Still, the very high prevalence of vitamin $\mathrm{D}$ deficiency worldwide along with its reported links to NCD make this an area of research with great potential impact for human health ${ }^{(1,8,19-22)}$.

At present the role of vitamin D deficiency in the development of NCD remains unclear, and there is currently no general agreement on what constitutes 'optimal' vitamin D status, or on the need for public health measures to screen for and correct deficiency $^{(21-26)}$. Vitamin D status is assessed by measuring the concentration of calcidiol $(25(\mathrm{OH}) \mathrm{D})$ in plasma or serum, preferably using liquid chromatography with tandem MS (LCMS/MS $)^{(27)}$. Plasma $25(\mathrm{OH}) \mathrm{D}<50 \mathrm{nmol} / 1$ is generally agreed to indicate deficiency of vitamin $\mathrm{D}$, but there is no clear consensus on the threshold of adequacy of vitamin $\mathrm{D}$, especially in relation to its reported non-skeletal effects. Plasma $25(\mathrm{OH}) \mathrm{D}$ $\geq 50 \mathrm{nmol} / \mathrm{l}$ may be adequate for bone health, but $75-100 \mathrm{nmol} / \mathrm{l}$ is suggested as optimal for lowering risk of NCD and overall mortality ${ }^{(6,7,24-26)}$

The link between vitamin D status and risk of NCD could be due, at least in part, to effects on oxidative stress and antioxidant status ${ }^{(6,28-33)}$. Vitamin D reportedly has antioxidant properties, protecting cultured human endothelial cells and retinal cone cells from oxidative challenge and activating the protective Nrf2-KEAP1 antioxidant pathway in diabetic rats ${ }^{(6,29-32)}$. In human study, lower vitamin D status was found to associate with higher levels of oxidative stress biomarkers in obese children ${ }^{(33)}$ : median plasma malondialdehyde and nitrotyrosine concentrations were, respectively, 80 and $50 \%$ higher $(P<0.05)$ in obese children with $25(\mathrm{OH}) \mathrm{D}<50 \mathrm{nmol} / \mathrm{l}$, although it is noted that obesity associates independently with both lower vitamin D and higher oxidative stress ${ }^{(2,3,34)}$. Depleted antioxidant status predisposes to oxidative stress, and logistic regression analysis of data from a study of forty preeclampsia patients revealed a positive association between $25(\mathrm{OH}) \mathrm{D}$ levels and plasma total antioxidant capacity $(\beta=0.428, P<0.02)^{(35)}$. In addition, significantly lower plasma total antioxidant capacity was found in fifty-eight vitamin D-deficient chronic hepatitis patients compared with nondeficient patients with the same disease, and a significant inverse correlation $(r-0.426 ; P<0.02)$ was seen between levels of plasma $25(\mathrm{OH}) \mathrm{D}$ and lipid hydroperoxides ${ }^{(36)}$. In sixty-five elderly subjects with type 2 diabetes or impaired fasting glucose, $25(\mathrm{OH}) \mathrm{D}$ correlated inversely with two oxidative stress biomarkers in plasma, namely oxidised LDL (OxLDL: $r-0 \cdot 413$, $P<0.01)$ and advanced protein peroxidation products $(r-0.475, P<0.001)^{(37)}$. As yet, studies of oxidative stress and antioxidant status in relation to vitamin $\mathrm{D}$ in still-healthy subjects are scarce. Some evidence of inverse association between plasma 25(OH)D and levels of advanced protein oxidation products was seen in a study of 411 apparently healthy subjects aged 18-81 years, but many of the subjects were obese, hypertensive, elderly or were smokers, $60 \%$ of participants were revealed during the study to have elevated risk of or suffering from cardiometabolic disease, and overall the oxidative stress

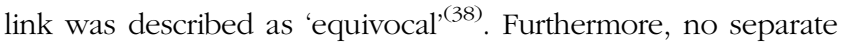
data on young adults were shown ${ }^{(38)}$. Therefore, we currently lack data on the putative vitamin D/oxidative stress association without the confounding effects of advanced age, obesity, smoking or pre-existing disease. This was the focus of the current study.

\section{Methods}

Fasting urine and heparinised venous blood samples were collected from 196 subjects (sixty-three males, 133 females). Antioxidants are more stable in heparin compared with EDTA plasma, and we regard heparinised plasma as the sample of choice $^{(39)}$. All participants reported being in good general health. None took regular medication, food or vitamin supplements. Participants did not smoke (all were 'never smokers') and were not obese. None had required medical treatment in the previous 6 months or been hospitalised within the previous year. All volunteers had been living in Hong Kong for at least 1 year. All but two (both of whom were of Caucasian ethnicity) were ethnic Chinese. Subject demographics were as follows: mean, age 20.8 (sD 1.6) years; weight 56.7 (sD .5) kg; height 163.3 (sD 8.8) cm; BMI 21.1 (sD 2.4) kg/m²: systolic blood pressure 113 (SD 10.0) $\mathrm{mmHg}$; diastolic blood pressure 64 (SD 7.7) $\mathrm{mmHg}$. This study was conducted according to the guidelines laid down in the Declaration of Helsinki and all procedures involving human subjects were approved by The Hong Kong Polytechnic University Human Subjects Ethics Subcommittee. Written informed consent was obtained from all subjects.

Samples were chilled immediately after collection. Heparinised blood plasma was harvested and aliquoted within $1 \mathrm{~h}$ of blood collection. Aliquots of centrifuged urine and plasma were stored at $-80^{\circ} \mathrm{C}$ and each aliquot was thawed once only for measurement. Plasma 25(OH)D was measured by LC-MS/MS, as previously described ${ }^{(9)}$. Three specific biomarkers of oxidative stress were measured: plasma allantoin, plasma OxLDL and urine 8-oxo-7,8-dihydro-2'-deoxyguanosine (8-oxodG). Allantoin and 8-oxodG were measured by LC-MS/MS, following published procedures ${ }^{(40,41)}$. Urine 8 -oxodG results are expressed as $\mathrm{nmol} / \mathrm{mmol}$ creatinine to compensate for differences in water content (i.e. urine concentration) of samples. Urine creatinine was measured by an automated colourimetric method on the day of sample collection. Plasma OxLDL was measured by immunoassay (Mercodia), and results are expressed as U/1 and as U/mmol LDL-cholesterol. Plasma total cholesterol (TC), HDL-cholesterol and TAG were measured by 
commercial kit methods on an AU480 analyzer (Beckman Coulter), and LDL-cholesterol concentration was calculated using the Friedewald equation as follows: LDL-cholesterol $=$ $(\mathrm{TC}-(\mathrm{HDL}-$ cholesterol $+\mathrm{Tg} / 2 \cdot 2))$ with all units in $\mathrm{mmol} / \mathrm{l}^{(42)}$. For antioxidant status, plasma non-enzymatic antioxidant capacity was measured by the ferric reducing antioxidant power (FRAP) assay ${ }^{(43,44)}$, and plasma ascorbic acid was measured using a validated modification of the FRAP assay, referred to as FRASC ${ }^{(44,45)}$. These were measured on the day of sample collection and as soon as possible after plasma separation. In addition, plasma FRAP value was corrected for uric acid content, which was done by subtracting $2 \times$ the plasma uric acid concentration from the FRAP value ${ }^{(43,46)}$. Uric acid was measured by an automated uricase kit method (Beckman Coulter). We also screened pure 25(OH)D to assess if it showed in vitro antioxidant activity. This was done by adding a drop of pure $25(\mathrm{OH}) \mathrm{D}$ calibrator (as used for the LCMS/MS analysis, and which was 3 PLUS $^{\circledR}$ 25-OH-Vitamin D3/D2 purchased from Chromosystems (Chromosystems)) to $1 \mathrm{ml}$ of freshly prepared FRAP assay reagent. The within-run and between run CV for 25(OH)D, 8-oxodG, allantoin and OxLDL were all $<4$ and $<7 \%$ respectively; for all other biomarkers the within-run and between-run $\mathrm{CV}$ were, respectively, $<1.5$ and $<2.0 \%$. The limit of detection (LoD) of each method was in every case below the values found in the test samples. Based on our in-house method evaluation and on the manufacturers' information for the commercial methods used, LoD were as follows: $25(\mathrm{OH}) \mathrm{D} \quad 11 \mathrm{nmol} / \mathrm{l}$; FRAP, $5.0 \mu \mathrm{mol} / 1$, ascorbic acid $3.0 \mu \mathrm{mol} / 1$, allantoin $0.09 \mu \mathrm{mol} / 1$, 8-oxodG $2.3 \mathrm{nmol} / \mathrm{l}$, OxLDL $0 \cdot 6 \mathrm{U} / 1$.

\section{Statistical analysis}

Data analysis was performed using Graphpad Prism 5. Data were tested by Kolmogorov-Smirvov test with DallalWilkinson-Lilliefors' $P$ for normal distribution. Results are presented as means and standard deviations, medians and range (low to high). Associations between plasma 25(OH)D and biomarkers of oxidative stress and antioxidant status were investigated using Pearson's or Spearman's correlation, as appropriate for distribution. Biomarker values across quartiles of plasma $25(\mathrm{OH}) \mathrm{D}$ were compared, and differences across $25(\mathrm{OH}) \mathrm{D}$ categories of $<25$ (which we regarded as severely deficient), $\geq 25<50$ and $\geq 50 \mathrm{nmol} / 1$ were explored using one-way ANOVA (with Newman-Kuels post hoc test) or Kruskal-Wallis test (with Dunns post hoc test), as appropriate. A $P<0.05$ was considered statistically significant. Power calculations were performed using data collected in our previous studies on young, apparently healthy subjects, and performed using PS Power and Sample Size Calculations Version 3.0 ${ }^{(47)}$ and revealed that forty-six subjects in each quartile of $25(\mathrm{OH}) \mathrm{D}$ is sufficient to be able to detect a true difference of at least $+/-0.35 \mu \mathrm{mol} / 1$ in allantoin, $+/-2.0 \mathrm{U} / \mathrm{mmol}$ in OxLDL: $\mathrm{LDL},+/-0.36$ in urine 8 -oxodG (as $\mathrm{nmol} / \mathrm{mmol}$ creatinine), $+/-94 \mu \mathrm{mol} / 1$ in FRAP, and $+/-10 \mu \mathrm{mol} / 1$ in ascorbic acid, with $80 \%$ power and a two-sided $P<0.05$. We hypothesize that these moderate effect sizes have meaningful clinical implications.

\section{Results}

Descriptive statistics for the biomarker results are shown in Table 1 . We note that the plasma $25(\mathrm{OH}) \mathrm{D}$ data, which showed a very high prevalence $(72 \%)$ of deficiency $(25(\mathrm{OH}) \mathrm{D}<50 \mathrm{nmol} / \mathrm{l})$ were reported previously ${ }^{(9)}$. Worth highlighting is the finding that only 2/196 young, still-healthy subjects were found to have plasma $25(\mathrm{OH}) \mathrm{D}$ in the suggested ${ }^{(24-26)}$ 'optimal' range of $75-100 \mathrm{nmol} / \mathrm{l}$. A weak direct association was found between plasma $25(\mathrm{OH}) \mathrm{D}$ concentration and the FRAP value, and also with the FRAP value corrected for uric acid: $r 0.252$ and 0.144 , respectively $(P<0.05$ for both). Pure 25(OH)D did not show detectable in vitro antioxidant activity in the FRAP assay. No significant association was seen between plasma $25(\mathrm{OH}) \mathrm{D}$ and any of the other biomarkers of interest. In regard to differences across quartiles of plasma $25(\mathrm{OH}) \mathrm{D}$, lower FRAP value $(P<0.05)$ was found in Q1 (the lowest quartile) compared with that in Q3 and in Q4 (Table 2), with FRAP values averaging, respectively, 10 and $14 \%$ higher in Q3 and Q4 compared with Q1. The FRAP values corrected for uric acid were 8, 10 and 15\% higher in Q2, Q3, Q4, respectively, compared with Q1, but the differences did not reach statistical significance. No significant differences across quartiles were seen in ascorbic acid, allantoin, OxLDL or 8-oxodG (Table 2). Comparing results across $<25, \geq 25<50$ and $\geq 50 \mathrm{nmol} / 125(\mathrm{OH}) \mathrm{D}$ groups, the mean FRAP value in those with $25(\mathrm{OH}) \mathrm{D} \geq 50 \mathrm{nmol} / \mathrm{l}$ was 1088 (sD 218) $\mu \mathrm{mol} / \mathrm{l}$, significantly higher than the FRAP values in the $25(\mathrm{OH}) \mathrm{D}$ groups of $<25$ and $\geq 25<50 \mathrm{nmol} / \mathrm{l}$, which were 950 (sD 206) and 1017 (sD 190) $\mu \mathrm{mol} / \mathrm{l}$, respectively. The FRAP value corrected for uric acid showed an increasing trend across the 25(OH)D groups: mean values from lowest to highest $25(\mathrm{OH}) \mathrm{D}$ groups were 346 (sD 108), 384 (SD 120) and 411 (sD 107) $\mu \mathrm{mol} / 1$, but the differences were not statistically significant. No significant differences were seen in ascorbic acid, allantoin, OxLDL or 8-oxodG across the 25(OH)D groups (data not shown).

\section{Discussion}

Vitamin D has long been established as essential for bone health, but data are accumulating on its pleiotropic effects and on association between low vitamin D status and increased risk

Table 1. Biomarker results on 196 healthy, non-obese, non-smoking subjects aged $18-26$ years*

(Mean values and standard deviations; medians and ranges)

\begin{tabular}{|c|c|c|c|c|}
\hline & Mean & SD & Median & Range \\
\hline Plasma 25(OH)D (nmol/l) & $42 \cdot 1$ & $13 \cdot 0$ & $40 \cdot 4$ & $15 \cdot 7-86 \cdot 8$ \\
\hline Plasma FRAP value $(\mu \mathrm{mol} / \mathrm{l})$ & 1033 & 202 & 1010 & $614-1666$ \\
\hline $\begin{array}{l}\text { Plasma FRAP corrected for } \\
\text { uric acid }(\mu \mathrm{mol} / \mathrm{l})\end{array}$ & 389 & 117 & $381 \cdot 0$ & $108-1089$ \\
\hline Plasma ascorbic acid $(\mu \mathrm{mol} / \mathrm{l})$ & $55 \cdot 3$ & $22 \cdot 0$ & 54.6 & $8 \cdot 5-115 \cdot 0$ \\
\hline Plasma allantoin $(\mu \mathrm{mol} / /)$ & 0.85 & 0.58 & 0.71 & $0.25-4.58$ \\
\hline Plasma OxLDL (Ü/l) & $36 \cdot 5$ & 9.9 & $36 \cdot 3$ & $17 \cdot 9-77.3$ \\
\hline $\begin{array}{l}\text { Plasma OxLDL:LDL-cholesterol } \\
\text { (U/mmol) }\end{array}$ & $13 \cdot 7$ & 3.51 & $13 \cdot 4$ & $4 \cdot 5-25 \cdot 1$ \\
\hline $\begin{array}{l}\text { Urine 8-oxodG (nmol/mmol } \\
\text { creatinine) }\end{array}$ & $1 \cdot 15$ & 0.76 & 0.98 & $0.32-7.48$ \\
\hline
\end{tabular}

25(OH)D, 25-hydroxycholecalciferol; FRAP, ferric reducing antioxidant power; OxLD, oxidised LDL; 8-oxodG, 8-oxo-7,8-dihydro-2'-deoxyguanosine.

* For allantoin, $n$ 195; for OxLDL, $n 185$. 
Table 2. Antioxidant and oxidative stress biomarker results in healthy young adults across quartiles $(\mathrm{Q})$ of plasma 25 -hydroxycholecalciferol $(25(\mathrm{OH}) \mathrm{D})$ concentrationt

(Mean values and standard deviations)

\begin{tabular}{|c|c|c|c|c|c|c|c|c|}
\hline & \multicolumn{2}{|c|}{ Q1 (n 49) } & \multicolumn{2}{|c|}{ Q2 (n 49) } & \multicolumn{2}{|c|}{ Q3 (n 49) } & \multicolumn{2}{|c|}{ Q4 (n 49) } \\
\hline & Mean & $S D$ & Mean & SD & Mean & SD & Mean & SD \\
\hline $25(\mathrm{OH}) \mathrm{D}(\mathrm{nmol} / \mathrm{l})$ & $27 \cdot 0$ & 4.7 & $36.5^{\star}$ & $2 \cdot 0$ & $45 \cdot 3^{*}$ & $3 \cdot 3$ & $59.7^{\star}$ & $7 \cdot 2$ \\
\hline Allantoin $(\mu \mathrm{mol} / \mathrm{l})$ & 0.89 & 0.77 & 0.80 & 0.50 & 0.92 & 0.59 & 0.79 & 0.38 \\
\hline OxLDL (U/l) & $36 \cdot 6$ & 9.04 & $37 \cdot 0$ & 9.60 & $34 \cdot 3$ & 9.83 & $38 \cdot 2$ & 11.04 \\
\hline OxLDL:LDL-cholesterol (U/mmol) & 13.92 & 3.08 & 13.56 & $3 \cdot 12$ & 13.43 & 4.19 & 13.84 & 3.65 \\
\hline 8-oxodG ( $\mathrm{nmol} / \mathrm{mmol}$ creatinine) & 1.24 & 0.65 & 0.99 & 0.57 & $1 \cdot 16$ & 1.02 & $1 \cdot 20$ & 0.71 \\
\hline FRAP $(\mu \mathrm{mol} / \mathrm{l})$ & 966 & 176 & 1005 & 187 & $1061^{*}$ & 209 & $1098^{*}$ & 213 \\
\hline FRAP corrected for uric acid $(\mu \mathrm{mol} / \mathrm{l})$ & 358 & 93 & 388 & 146 & 394 & 105 & 414 & 112 \\
\hline Ascorbic acid $(\mu \mathrm{mol} / \mathrm{l})$ & $58 \cdot 1$ & $25 \cdot 2$ & 53.4 & $24 \cdot 0$ & 51.4 & $17 \cdot 2$ & 59.9 & $23 \cdot 3$ \\
\hline
\end{tabular}

OxLD, oxidised LDL; 8-oxodG, 8-oxo-7,8-dihydro-2'-deoxyguanosine; FRAP, ferric reducing antioxidant power.

* $P<0.05$ compared with the value in $Q 1$.

† For OxLDL $n /$ quartile $=46$.

of cancer, CVD, diabetes and other NCD, as well as all-cause mortality ${ }^{(6,7,10-20)}$. Vitamin D deficiency reportedly associates with increased oxidative stress in the elderly, the obese, and in those suffering from diabetes, severe asthma and various other diseases $^{(33-38,48)}$. However, no association was seen in stroke patients, the results of some studies are equivocal, and deficiency of vitamin $\mathrm{D}$ could be a side-effect or consequence of preexisting disease ${ }^{(23,38,49)}$. Still, the reported associations between vitamin D deficiency and increased oxidative stress, and between vitamin D deficiency and increased risk of NCD, combine to make a compelling case for this as an area of high research interest and potential impact on human health and public health nutritional policy because: (a) NCD pose the greatest threat to global health and economic well-being ${ }^{(1,5,50)}$, (b) oxidative stress is a major player in the development of $\mathrm{NCD}^{(1-4)}$ and (c) deficiency of vitamin D is highly prevalent worldwide, even in young, apparently healthy people living in sunny regions ${ }^{(8,9,20-22)}$. Indeed, in regard to this last point, it has been estimated $^{(20)}$ that around one billion people across all regions, ethnicities and ages lack sufficient vitamin D. If a direct link to oxidative stress is confirmed, deficiency of vitamin $\mathrm{D}$ has important implications far beyond musculoskeletal health. Our focus in this study was on the vitamin D/oxidative stress association, using a biomarker approach. Previous reports of significant association are interesting, but until now data are lacking from subjects who are free from the various other factors that drive oxidative stress, such as advanced age, obesity, smoking and pre-existing disease ${ }^{(1-4)}$. This lack was addressed in this study of healthy, non-obese, non-smoking young adults. Plasma $25(\mathrm{OH}) \mathrm{D}$ was measured specifically using LC-MS/MS, and several sensitive and specific biomarkers of oxidative stress and antioxidant status were measured. It is noted that the panel of biomarkers used in this study was fairly comprehensive, and covered biomarkers for water soluble antioxidant status (total FRAP, FRAP corrected for uric acid and ascorbic acid) that could be expected to be depleted if oxidative stress was increased, as well as oxidative stress biomarkers that reflect pro-oxidant change in the aqueous system (allantoin and 8-oxodG) and the lipophilic system (OxLDL) $)^{(4,40,41,43-45)}$.

A significant correlation was seen between $25(\mathrm{OH}) \mathrm{D}$ and total antioxidant status (as the FRAP value) and between 25(OH)D and
FRAP corrected for uric acid, though it is noted that the associations were weak. No significant correlation was seen between plasma $25(\mathrm{OH}) \mathrm{D}$ and any of the other biomarkers of interest. Lower total antioxidant status was seen in those with lower vitamin D status but, whereas statistically significant, the difference was not large, and no significant differences in biomarkers of oxidative stress were seen across quartiles of plasma 25(OH)D or across different vitamin D status groups. Depleted antioxidant status increases the likelihood of an increase in oxidation-induced damage to biomolecules, and the findings in relation to vitamin D and FRAP and FRAP corrected for uric acid are interesting. However, the results overall do not support a strong underlying link between vitamin D deficiency and increased oxidative stress in young, still-healthy, non-obese subjects. It is noted also that no association with vitamin D status was seen in our study of oxidation-induced DNA damage ${ }^{(51)}$.

Antioxidant/oxidative stress balance is affected by many factors $^{(3,4)}$. Habitual diet is one important factor, and others include cigarette smoking, obesity, inflammation, food supplements and presence of disease. It is noted that our 196 subjects were not obese, did not smoke, none were taking any kind of food or vitamin supplements or medicines, and all reported being in good general health. It is noted also that the contribution of diet to vitamin D status is generally minor ${ }^{(6,21,22)}$. None of our volunteers reported being vegetarian or having special dietary requirements, but we did not do a detailed dietary survey, and dietary factors could have confounded results. However, the influence of diet, even if known in detail, cannot be compensated for in any easy manner. Issues of limited bioavailability, excretion and utilisation of dietaryderived antioxidants mean that there is a very complex relationship between dietary input and measured levels of antioxidant status biomarkers such as FRAP and ascorbic acid. It is noted that diet does not directly influence the biomarkers used to assess oxidative stress in fasting samples in this study, namely 8 -oxodG, allantoin and $\operatorname{OxLDL}^{(4,40,41,52)}$. These oxidative stress biomarkers are of endogenous, not dietary origin, as are their non-oxidised forms (LDL, nucleobases and uric acid) and while intake of food and postprandial oxidative stress could affect these biomarkers acutely, it is noted that all samples 
collected were fasting samples. Still, if overall antioxidant status is 'good' due to the combined effect of all endogenous and exogenous antioxidants, then it is possible to speculate that, in this situation, the antioxidant effect of vitamin D (if one exists) could be mitigated, removing any obvious links between vitamin D status and oxidative stress.

It is noted that most (>70\%) of the 196 young, otherwise healthy subjects studied here were deficient in vitamin $\mathrm{D}$ when $25(\mathrm{OH}) \mathrm{D}<50 \mathrm{nmol} / \mathrm{l}$ was used to define deficiency. When using the suggested threshold of $75 \mathrm{nmol} / 1$ only two subjects had adequate vitamin $D$. This highlights the very high prevalence of vitamin $\mathrm{D}$ deficiency in young adults. In this study, samples were collected year-round. In contrast to findings in Northern European countries showing a strong seasonal influence on vitamin D status we and others have found no significant seasonal differences in vitamin D status in Hong Kong, which lies $22^{\circ} \mathrm{N}$. A local study reported on determinants of serum 25(OH)D in 2694 subjects aged from 6 to over 65 years and, whereas very limited $25(\mathrm{OH}) \mathrm{D}$ data were shown, overall mean values were similar across the seasons ${ }^{(53)}$. Therefore, season is not the main determinant of vitamin D status in Hong Kong. The main determinants, as revealed by $\mathrm{Xu}$ et $a l .^{(53)}$, are duration of exposure to sunshine and vitamin D-rich food/supplementation intake. The very high prevalence of deficiency is due, we suggest, to the largely indoor lifestyle of our young adults, coupled with a commonly observed avoidance of direct sun exposure in many local residents. Furthermore, there is no local policy of mandatory food fortification with vitamin $\mathrm{D}$. The results of this study also highlight the difficulties in clarifying the putative link between vitamin D deficiency, oxidative stress and risk of NCD in a general population, because few members have $25(\mathrm{OH}) \mathrm{D}$ $>50 \mathrm{nmol} / 1$, and those with values of $75 \mathrm{nmol} / 1$ or greater are very hard to find. To more fully investigate the association between vitamin D and oxidative stress, the study of effects of correction of vitamin D deficiency on oxidative stress and antioxidant status biomarkers in deficient but otherwise healthy subjects is needed.

\section{Acknowledgements}

The authors thank Dr WY Chung and Dr M Devaki for their skilled technical assistance.

This work was financially supported by the Research Grants Council (RGC) of the University Grants Committee of Hong Kong (General Research Fund Grant PolyU 560113) and by The Hong Kong Polytechnic University. The RGC and The Hong Kong Polytechnic University had no role in the design, analysis or writing of this article.

All authors were involved in formulating the research questions and designing the study. E. W. W, I. F. F. B, M. Y. P. and J. W. were involved in recruiting volunteers. E. W. W. and I. F. F. B. were responsible for sample handling and testing, and for drafting the paper. E. W. W., I. F. F. B. and P. M. S. were responsible for statistical analysis. All authors were involved in refining and finalising the paper. All authors approve the submission.

The authors declare that there are no conflicts of interest.

\section{References}

1. World Health Organization (2013) Global Action Plan for the Prevention and Control of Noncommunicable Diseases 2013-2020. Geneva: WHO.

2. Camps J \& García-Heredia A (2014) Introduction: oxidation and inflammation, a molecular link between noncommunicable diseases. Adv Exp Med Biol 824, 1-4.

3. Rani V, Deep G, Singh RK, et al. (2016) Oxidative stress and metabolic disorders: pathogenesis and therapeutic strategies. Life Sci 148, 183-193.

4. Halliwell B \& Gutteridge JMC (2007) Free Radicals in Biology and Medicine, 4th ed. Oxford: Oxford University Press.

5. Global Health (2013) Investing in Our Future: NonCommunicable Disease. Washington, DC: Global Health. http:// www.globalhealth.org/wp-content/uploads/GlobalHealthBriefing Book_FINAL_web.pdf

6. Christakos S, Dhawan P, Verstuyf A, et al. (2016) Vitamin D: metabolism, molecular mechanism of action, and pleiotropic effects. Physiol Rev 96, 365-408.

7. Schottker B, Haug U, Schomburg L, et al. (2013) Strong association of hydroxyvitamin D concentrations with all-cause, cardiovascular, cancer and respiratory disease mortality in a large cohort study. Am J Clin Nutr 97, 782-793.

8. Hilger J, Friedel A, Herr R, et al. (2014) A systematic review of vitamin D status in populations worldwide. Br J Nutr 111, 23-45.

9. Wang EW, Pang MYC, Siu PPM, et al. (2016) Vitamin D status and cardiometabolic risk factors in young adults in Hong Kong: associations and implications. APJCN (Epublication ahead of print version).

10. Skaaby T (2015) The relationship of vitamin D status to risk of cardiovascular disease and mortality. Dan Med J 62, pii B5008.

11. Mitri J, Muraru MD \& Pittas AG (2011) Vitamin D and type 2 diabetes: a systematic review. Eur J Clin Nutr 65, 1005-1015.

12. Norman PE \& Powell JT (2015) Vitamin D and cardiovascular disease. Circ Res 114, 379-393.

13. Welsh P, Doolin O, McConnachie A, et al. (2012) Circulating 25OHD, dietary vitamin D, PTH, and calcium associations with incident cardiovascular disease and mortality: the MIDSPAN Family Study. J Clin Endocrinol Metab 97, 4578-4587.

14. Pilz S, Verheyen N, Grübler MR, et al. (2016) Vitamin D and cardiovascular disease prevention. Nat Rev Cardiol 13, 404-417.

15. Virtanen JK, Nurmi T, Voutilainen S, et al. (2011) Association of serum 25-hydroxvitamin $\mathrm{D}$ with the risk of death in a general older population in Finland. Eur $J$ Nutr 50, 305-312.

16. Jenab M, Bueno-de-Mesquita HB, Ferrari P, et al. (2010) Association between pre-diagnostic circulating vitamin D concentration and risk of colorectal cancer in European populations: a nested case-control study. BMJ 340, b5500.

17. Lim S, Kim MJ, Choi SH, et al. (2013) Association of vitamin D deficiency with incidence of type 2 diabetes in high-risk Asian subjects. Am J Clin Nutr 97, 524-530.

18. Gagnon C, Lu ZX \& Magliano DJ (2011) Serum 25-hydroxyvitamin D, calcium intake, and risk of type 2 diabetes after 5 years: results from a national populationbased prospective study (the Australian Diabetes, Obesity and Lifestyle study). Diabetes Care 34, 1133-1138.

19. Norman PE \& Powell JT (2014) Vitamin D and cardiovascular disease. Circ Res 114, 379-393.

20. Palacios C \& Gonzalez L (2014) Is vitamin D deficiency a major public health problem? J Steroid Biochem Mol Biol 144 , $138-145$. 
21. Balvers MGJ, Brouwer-Brolsma EM, Enderberg S, et al. (2015) Recommended intakes of vitamin D to optimise health, associated 25-hydroxyvitamin D concentrations, and dosing regimens to treat deficiency: workshop report and overview of current literature. J Nutr Sci $\mathbf{4}$, e23.

22. Battault S, Whiting SJ, Peltier SL, et al. (2013) Vitamin D metabolism, functions and needs: from science to health claims. Eur J Nutr 52, 429-441.

23. Autier P, Boniol M, Pizot C, et al. (2014) Vitamin D status and ill health: a systematic review. Lancet Diabetes Endocrinol 2, 76-89.

24. Heaney RP \& Holick MF (2011) Why the IOM recommendations for vitamin D are deficient. J Bone Mineral Res 26, 455-457.

25. Vieth R (2011) Why the minimum desirable serum 25-hydroxyvitamin D level should be $75 \mathrm{nmol} / \mathrm{l}(30 \mathrm{ng} / \mathrm{ml})$. Best Prac Res Clin Endocrinol Metab 25, 681-691.

26. Spedding S, Vanlint S, Morris H, et al. (2013) Does vitamin D sufficiency equate to a single serum 25-hydroxyvitamin D level or are different levels required for non-skeletal diseases? Nutrients 5, 5127-5139.

27. Farrell CJ, Martin S, McWhinney B, et al. (2012) State-of-the-art vitamin D assays: a comparison of automated immunoassays with liquid chromatography-tandem mass spectrometry methods. Clin Chem 58, 531-542.

28. Nikooyeh B \& Neyestani TR (2016) Oxidative stress, type 2 diabetes and vitamin D: past, present and future. Diabetes Metab Res Rev 32, 260-267.

29. Peng X, Vaishnav A, Murillo G, et al. (2010) Protection against cellular stress by 25 -hydroxy D3 in breast epithelial cells. J Cell Biochem 110, 1324-1333.

30. Uberti F, Lattuada D, Morsanuto V, et al. (2014) Vitamin D protects human endothelial cells from oxidative stress through the autophagic and survival pathways. J Clin Endocrinol Metab 99, 1367-1374

31. Tohari AM, Zhou X \& Shu X (2016) Protection against oxidative stress by vitamin D in cone cells. Cell Biochem Funct 34, 82-94.

32. George N, Kumar TP, Antony S, et al. (2012) Effect of vitamin $\mathrm{D}_{3}$ in reducing metabolic and oxidative stress in the liver of streptozotocin-induced diabetic rats. Br J Nutr 108, 1410-1418.

33. Codoñer-Franch $\mathrm{P}$, Tavárez-Alonso $\mathrm{S}$, Simó-Jordá $\mathrm{R}$, et al. (2012) Vitamin D status is linked to biomarkers of oxidative stress, inflammation, and endothelial activation in obese children. J Pediatr 161, 848-854.

34. Pereira-Santos M, Costa PR, Assis AM, et al. (2015) Obesity and vitamin D deficiency: a systematic review and metaanalysis. Obes Rev 16, 341-349.

35. Pourghassem Gargari B, Pourteymour Fard Tabrizi F, Sadien B, et al. (2016) Vitamin D status is related to oxidative stress but not high-sensitive C-reactive protein in women with pre-eclampsia. Gynecol Obstet Invest 81, 308-314.

36. De Almeida JP, Liberatti LS, Barros FE, et al. (2016) Profile of oxidative stress markers is dependent on vitamin D levels in patients with chronic hepatitis C. Nutrients 32, 362-367.

37. Gradinaru D, Borsa C, Ionescu C, et al. (2012) Vitamin D status and oxidative stress markers in elderly with impaired fasting glucose and type 2 diabetes mellitus. Aging Clin Exp Res 24, 595-602.
38. Krivošíková Z, Gajdoš M \& Šebeková K (2015) Vitamin D levels decline with rising number of cardiometabolic risk factors in healthy adults: association with adipokines, inflammation, oxidative stress and advanced glycation markers. PLOS ONE 10, e0131753.

39. Benzie IFF \& Chung WY (1999) Total antioxidant power of plasma: male-female differences and effect of anticoagulant used. Clin Chim Acta 36, 104-106.

40. Chung WY \& Benzie IF (2013) Plasma allantoin measurement by isocratic liquid chromatography with tandem mass spectrometry: method evaluation and application in oxidative stress biomonitoring. Clin Chim Acta 424, 237-244.

41. Lee KF, Chung WY \& Benzie IF (2010) Urine 8-oxo-7,8dihydro-2'-deoxyguanosine (8-oxodG), a specific marker of oxidative stress, using direct, isocratic LC-MS/MS: method evaluation and application in study of biological variation in healthy adults. Clin Chim Acta 411, 416-422.

42. Friedewald WT, Levy RI \& Fredrickson DS (1972) Estimation of the concentration of low-density lipoprotein cholesterol in plasma, without use of the preparative ultracentrifuge. Clin Chem 18, 499-502.

43. Benzie IF \& Strain JJ (1996) The ferric reducing ability of plasma (FRAP) as a measure of 'antioxidant power': the FRAP assay. Anal Biochem 239, 70-76.

44. Benzie IF \& Strain JJ (1999) Ferric reducing/antioxidant power assay: direct measure of total antioxidant activity of biological fluids and modified version for simultaneous measurement of total antioxidant power and ascorbic acid concentration. Methods Enzymol 299, 15-27.

45. Chung WY, Chung JK, Szeto YT, et al. (2001) Plasma ascorbic acid: measurement, stability and clinical utility revisited. Clin Biochem 34, 623-627.

46. Benzie IF \& Strain JJ (1996) Uric acid: Friend or foe? Redox Rep 2, 231-234.

47. Department of Biostatistics, Vanderbilt University School of Medicine (2014) PS: Power and Sample Size Calculation. biostat.mc.vanderbilt.edu (accessed August 2014).

48. Lan N, Luo G, Yang X, et al. (2014) 25-Hydroxyvitamin D3 deficiency enhances oxidative stress and corticosteroid resistance in severe asthma exacerbation. PLOS ONE (Epublication ahead of print version).

49. Afshari L, Amani R, Soltani F, et al. (2015) The relation between serum vitamin $\mathrm{D}$ levels and body antioxidant status in ischemic stroke patients: a case-control study. Adv Biomed Res $\mathbf{4}, 213$

50. World Economic Forum (2016) The global risks report 2016. www3.weforum.org/docs/Media/TheGlobalRisksReport 2016.pdf

51. Wang EW, Collins AR, Pang MYC, et al. (2016) Vitamin D and oxidation-induced DNA damage: is there a connection? Mutagen 31, 655-659.

52. Broedbaek K, Weimann A, Stovgaard ES, et al. (2011) Urinary 8-oxo-7,8-dihydro-2'-deoxyguanosine as a biomarker in type 2 diabetes. Free Radic Biol Med 51, 1473-1479.

53. Xu C, Perera RA, Chan YH, et al. (2015) Determinants of serum 25-hydroxyvitamin D in Hong Kong. Br J Nutr 114, 144-151. 